\title{
LA AYUDA HUMANITARIA DE LOS BRITISH QUAKERS DURANTE LA GUERRA CIVIL ESPAÑOLA (1936-1939): EL CASO DEL HOSPITAL INFANTIL DE POLOP DE LA MARINA (ALICANTE) ${ }^{1}$
}

\author{
Xavier García Ferrandis \\ Universidad Católica de Valencia "San Vicente Mártir» \\ xavier.garcia@ucv.es \\ ORCID iD: https://orcid.org/0000-0002-0575-6902 \\ Àlvar Martínez-Vidal \\ Instituto de Historia de la Medicina y de la Ciencia López Piñero, Universitat de València \\ alvar.martinez@uv.es \\ ORCID iD: https://orcid.org/0000-0001-9760-4449
}

Recibido: 7 septiembre 2017; Aprobado: 3 noviembre 2018.

Cómo citar este artículo/Citation: García Ferrandis, Xavier / Martínez-Vidal, Àlvar (2019), "La ayuda humanitaria de los British Quakers durante la Guerra Civil española (1936-1939): el caso del Hospital Infantil de Polop de la Marina (Alicante)", Asclepio, 71(1): p253. https://doi.org/10.3989/asclepio.2019.05

RESUMEN: Debido a su condición geoestratégica de retaguardia durante la Guerra Civil española, el País Valenciano se convirtió en una de las zonas republicanas que acogieron mayor número de refugiados, entre ellos muchos niños. El Estado republicano se mostró del todo incapaz de afrontar los retos derivados de esta crisis demográfica y sanitaria e hizo un llamamiento en busca de ayuda. Una de las primeras agencias humanitarias transnacionales en responder fue la Religious Society of Friends del Reino Unido, más conocidos como el Friends Service Committee o simplemente los Quakers, una comunidad religiosa disidente fundada en Inglaterra en el siglo XVII. Durante la Guerra Civil, los cuáqueros impulsaron numerosas iniciativas de carácter humanitario en los dos bandos enfrentados, habilitando colonias agrícolas, talleres, cantinas y hospitales.

Este trabajo analiza en profundidad el hospital infantil que los Quakers habilitaron en Alicante en septiembre de 1937 y que posteriormente fue trasladado al municipio de Polop de la Marina. Nos centraremos en aspectos tales como la ubicación y administración del centro sanitario, el tipo de pacientes que allí se atendieron, el personal sanitario que allí trabajó, la evolución del hospital a lo largo de la guerra y su recorrido tras la victoria franquista, así como la motivación que impulsó a los voluntarios británicos a promover ese proyecto y a llevarlo a cabo. Asimismo, reconstruiremos la figura y la trayectoria de Manuel Blanc Rodríguez (1899-1971), un pediatra desconocido por la historiografía, que asumió la dirección de ese hospital británico.

PALABRAS CLAVE: Guerra Civil española; cuáqueros británicos; infancia; refugiados; hospital; Alicante; Polop de la Marina; Manuel Blanc Rodríguez.

\section{HUMANITARIAN AID FROM THE BRITISH QUAKERS DURING THE SPANISH CIVIL WAR (1936-1939): THE CASE OF THE CHILDREN'S HOSPITAL OF POLOP DE LA MARINA (ALICANTE)}

ABSTRACT: During the Spanish Civil War, the Valencian Country became one of the republican zones receiving greater number of refugees, due to its rearguard geostrategic condition. Among them there were many children. The Republican State was totally unable to face the challenges of this demographic and health crisis and appealed for help. The British Religious Society of Friends (also known as the Friends Service Committee or Quakers) was one of the first transnational humanitarian agencies to respond. They are a dissident religious community founded in England in the 17th century. During the Spanish Civil War, the Quakers encouraged many humanitarian initiatives on both sides in conflict, as agricultural colonies, workshops, canteens and hospitals.

This paper analyzes in depth the children's hospital that the Quakers enabled in Alicante in September 1937, later transferred to the village of Polop de la Marina. We will focus on aspects such as the location and administration of the health center, the type of patients that were attended, the health personnel who worked, the evolution of the hospital throughout the war and what happened to it after the Francoist victory. We will also stress the motivation of the British volunteers to promote and carry out this project. Finally, we will reconstruct the career of Manuel Blanc Rodríguez (1899-1971), the pediatrician who assumed the management of that British hospital. His figure remains unknown by the historiography.

KEY WORDS: Spanish Civil War; British Quakers; childhood; refugees; hospital; Alicante; Polop de la Marina; Manuel Blanc Rodríguez. Copyright: @ 2019 CSIC. Este es un artículo de acceso abierto distribuido bajo los términos de la licencia de uso y distribución Creative Commons Reconocimiento 4.0 Internacional (CC BY 4.0). 


\section{INTRODUCCIÓN}

En el caso de la Guerra Civil española, a la carestía padecida por la población civil se sumó el bombardeo aéreo y naval de las ciudades republicanas. Ante esta situación, el Gobierno republicano optó por trasladar a los refugiados a ciudades de retaguardia, como Valencia o Barcelona, siendo el contingente de niños el más numeroso ${ }^{2}$. Esta medida con frecuencia supuso la dispersión del núcleo familiar y, en paralelo, la necesidad de dar acogida institucional a la infancia refugiada para satisfacer sus necesidades, a saber, educación, asistencia sanitaria y alimentación, además de alojamiento en condiciones adecuadas.

La problemática de los niños refugiados en la guerra de España adquirió una gran proporción debido a la evolución militar y política de la contienda. Efectivamente, con cada victoria franquista el territorio de la República iba reduciéndose y, paradójicamente, iba recibiendo mayor número de refugiados. Las sucesivas derrotas sufridas por el ejército republicano hicieron que el Gobierno se viera desbordado por un problema cuya magnitud se acrecentaba de día en día (Fernández Soria, 1987, p. 88).

Con el fin de suplir las insuficiencias de los organismos oficiales, numerosas organizaciones humanitarias tomaron la iniciativa de socorrer a la población civil más necesitada. La ayuda que se destinó a la España republicana provenía de países democráticos -Francia, Reino Unido, EUA- cuyos gobiernos habían suscrito el Pacto de No Intervención (Alted Vigil, 2003, p. 51). También se canalizó ayuda humanitaria, procedente de dichos países, hacia la España franquista, que respondía estrictamente a principios de independencia e imparcialidad: entre otras agencias, Save the Children International Union, el American Friends Service Committee (cuáqueros norteamericanos) y el Comité Internacional de la Cruz Roja (Pretus, 2015). Cabe apuntar que la zona nacional, además, recibió ayuda de voluntarios extranjeros, entre ellos rusos blancos, rumanos de la Guardia de Hierro y católicos ingleses e irlandeses, que se sentían plenamente identificados con la causa de los sublevados (Keene, 2001). En cualquier caso, la Guerra de España constituye, entre las dos guerras mundiales, un excelente laboratorio para estudiar la eclosión, el desarrollo y la actuación de numerosas organizaciones privadas dedicadas al socorro de las poblaciones civiles, en especial de los niños, víctimas de un conflicto que movilizó personal y recursos de numerosos países
(Farré, 2014, pp. 87-119). Una de las primeras organizaciones en responder a la crisis humanitaria derivada del estallido de la guerra fue la Religious Society of Friends del Reino Unido, más conocidos como el Friends Service Committee o simplemente los Quakers. La historiografía sobre la ayuda humanitaria dispensada por los British Quakers durante la contienda española se ha incrementado notablemente en los últimos años. Al trabajo ya clásico de Jim Fyrth (Fyrth, 1986), hay que sumar aportaciones más recientes y centradas en diferentes ámbitos, como el caso concreto de Cataluña (Serra Sala, 2006), la colaboración de los cuáqueros con otros agentes humanitarios (Kershner, 2011; Palfreeman, 2014) o el fundamento religioso de sus iniciativas solidarias (Mendlesohn, 2002). Finalmente, en fecha muy reciente, han sido publicadas dos monografías que contienen abundante información sobre la ayuda humanitaria de los cuáqueros británicos durante la Guerra de España (Derby, 2015; Pretus, 2015).

Sin embargo, la historiografía revisada comparte dos carencias que justifican nuestra aportación. Desde el punto de vista metodológico, tan solo una de ellas analiza fuentes depositadas en los archivos de las localidades españolas donde se establecieron los British Quakers; en segundo lugar, la bibliografía secundaria señalada se centra en la actuación de los voluntarios británicos en Cataluña, Almería y Murcia. Las razones son obvias: para el caso catalán, la cercanía de la frontera y la relativa tranquilidad durante gran parte de la guerra posibilitaron la implantación de numerosos mecanismos de protección a la infancia; para los casos de Almería y Murcia, la llegada masiva de miles refugiados tras la caída de Málaga en febrero de 1937.

Sin embargo, la bibliografía crítica consultada trata con menor intensidad los pormenores del trabajo de los cuáqueros británicos en Alicante, una zona -al igual que las anteriores- situada en la retaguardia republicana durante la totalidad del conflicto $y$, por tanto, altamente receptiva a acoger toda suerte de iniciativas de carácter humanitario para acoger a la población refugiada. Efectivamente, Alicante absorbió en un segundo tiempo gran parte del aluvión de los refugiados malagueños, tras pasar estos por Almería y Murcia, así como grandes contingentes de población infantil evacuada de zonas de conflicto, muy especialmente de Madrid.

El objetivo principal de nuestro trabajo es analizar la acción humanitaria -muy especialmente la vertien- 

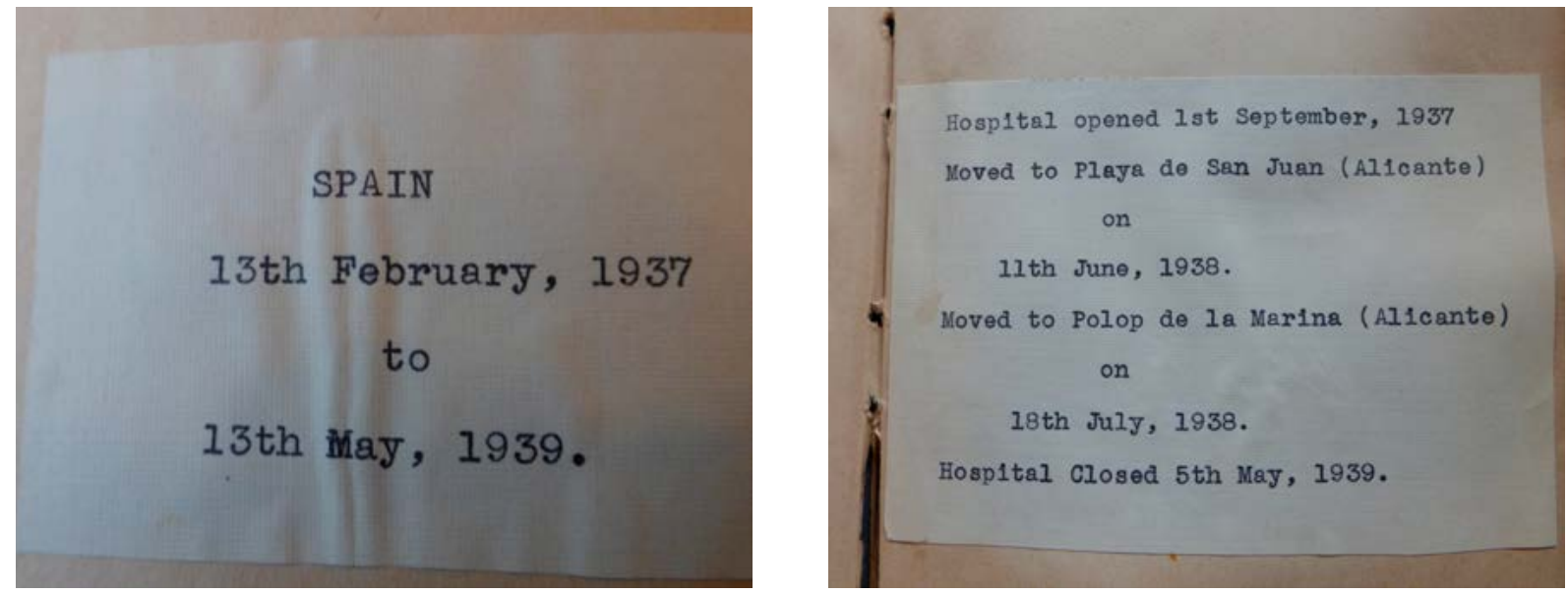

Imagen 1. El álbum de Mary Elmes contiene numerosas fotografías que reflejan la labor humanitaria de los cuáqueros en la España republicana (izquierda). El apartado dedicado al Hospital de Polop de la Marina es especialmente amplio (derecha). Fuente: archivo personal de Mary Elmes.

te sanitaria- desarrollada por los cuáqueros británicos en Alicante; en concreto, abordaremos en profundidad el Hospital Infantil de Polop de la Marina. Asimismo, analizaremos la identidad y la trayectoria, así como el contexto en el que Manuel Blanc Rodríguez desarrolló su labor como director médico del hospital infantil.

La metodología utilizada para alcanzar los objetivos propuestos se basa en el análisis y cotejo de documentos custodiados en la Friends House Library (Londres) y en la Marx Memorial Library (Londres), aunque también hemos consultado de manera tangencial documentos depositados en el archivo de la familia Young (Londres) y en la National Library of New Zealand (Wellington, Nueva Zelanda). Asimismo, se han realizado búsquedas en los archivos municipales de las localidades de la provincia de Alicante donde se tiene constancia de la presencia de los cuáqueros británicos y sus colaboradores durante la guerra (Crevillent, Benidorm, Elx y Alicante ciudad). Por otra parte, utilizaremos fuentes orales para intentar localizar el edificio que albergó el hospital en Polop de la Marina, así como para determinar si en el municipio persiste algún recuerdo del paso de las voluntarias británicas.

Por otra parte, expondremos material fotográfico perteneciente al archivo personal de Mary Elmes, una voluntaria irlandesa que durante la Guerra Civil española trabajó en varias iniciativas humanitarias impulsadas por los cuáqueros británicos y que, como se verá posteriormente, dirigió el Hospital de Polop de la Marina. Se trata de las fotografías correspondientes -seis en total- a este centro contenidas en un álbum familiar (ver imagen 1), propiedad de sus hijos Patrick y Caroline Danjou-Elmes. No pretendemos aquí analizar dichas fotografías, ni contextualizarlas en la cultura visual del movimiento humanitario durante la Guerra Civil; se pretende más bien acercar al lector a algunos de los escenarios descritos y mostrar el aspecto de las protagonistas de los hechos que se narran a continuación.

\section{UNA APROXIMACIÓN A LA AYUDA SANITARIA TRANSNACIONAL A LA ESPAÑA REPUBLICANA DURANTE LA GUERRA CIVIL}

Cuando la Guerra Civil estalló, se hizo necesaria la reorganización de los servicios sanitarios, tanto en los frentes de guerra como en la retaguardia. Ante la incapacidad del gobierno de la República para acometer en solitario este reto, el 26 de julio de 1936, hizo una llamada mundial para recibir ayuda. Una de las primeras agencias humanitarias en responder fue el Friends Service Council del Reino Unido, más conocidos como Quakers (cuáqueros en castellano). Como es conocido, el cuaquerismo es una doctrina religiosa disidente de la Iglesia anglicana fundada por George Fox (1624-1691). Entre la trayectoria histórica de los Quakers cabe destacar el antiesclavismo, el antibelicismo, la objeción de conciencia y la asistencia sanitaria en conflictos bélicos, tanto en primera línea como en la retaguardia (Van Etten, 1938, pp. 11-12; Kershner, 2011, pp. 7-15). Desde 1931 existía un proyecto del Friends Service Council (a partir de ahora 
FSC), de crear en España un centro cuáquero internacional. Con esta intención y al amparo de la libertad de culto que ofrecía la Constitución republicana, en 1936 el matrimonio integrado por los cuáqueros británicos Alfred y Norma Jacob se trasladaron a Espa$\tilde{n ̃ a}{ }^{3}$. El golpe militar de julio alteró profundamente el proyecto inicial, priorizando la ayuda humanitaria. En un primer momento la labor del FSC se centró en Catalunya, un territorio alejado de los frentes de guerra y que empezaba a recibir grandes contingentes de población refugiada.

Además de en Cataluña, el FSC centró sus esfuerzos en Almería, Murcia y Alicante habilitando y gestionando varios hospitales infantiles. EI FSC intentaba así dar una respuesta asistencial a la crisis demográfica y humanitaria que se produjo tras la entrada en Málaga de las tropas italianas el 8 de febrero de 1937. Efectivamente, la caída de Malága provocó uno de los mayores éxodos y crímenes contra la población civil de la Guerra Civil española (Bethune, 1937; Bethune, 2012; Majada Neila, 2014). Menos conocido que el éxodo que supuso la Retirada y que el crimen cometido en Gernika ${ }^{4}$, la estampida humana a lo largo de la carretera Málaga-Almería afectó a unas 100.000 personas, de las que más de la mitad eran mujeres y niños y entre las que se encontraban 40.000 soldados republicanos en retirada. Muchos refugiados malagueños continuaron su exilio hasta Valencia, Barcelona y llegaron a la frontera francesa. Así, sabemos que de los 600 refugiados que había en 1937 en Puigcerdà y su comarca (La Cerdanya, Girona) 264 procedían de Málaga y su provincia ${ }^{5}$. Durante el éxodo muchas familias malagueñas habían sufrido la dispersión de su miembros, por lo que en Puigcerdà se articularon mecanismos para su reagrupamiento, como las noticias en publicaciones periódicas ${ }^{6}$. Los campos del sur de Francia fueron el destino final de muchos refugiados malagueños ${ }^{7}$.

La primera respuesta de carácter humanitario vino de la mano de Sir George Young (1872-1952), aristócrata, diplomático y filántropo británico. En estrecha colaboración con el FSC, Young fundó un hospital infantil en Almería (Palfreeman, 2014, pp. 130-159). Con una capacidad para 25 niños, abrió sus puertas el 21 de febrero de 1937, tan solo unos días después de la caída de Málaga. En un primer momento, la financiación del hospital de Almería corrió a cargo de Young y de los cuáqueros británicos, cuyos recursos, sin embargo, eran limitados. Por ello a finales de 1937, los cuáqueros norteamericanos (American
Friends Service Committe o AFSC) asumieron la gestión del hospital a través de la trabajadora social Esther Farquhar $^{8}$.

En abril de 1937, la colaboradora del FSC Francesca Wilson llegó a Murcia9. Como consecuencia de la caída de Málaga dos meses antes y saturada Almería, la ciudad del Segura acogía a miles de refugiados en varios refugios en condiciones infrahumanas (hacinamiento, falta de alimentos y de higiene...). Muchos de ellos eran niños que necesitaban asistencia sanitaria, pero el Hospital Provincial de Murcia estaba colapsado por la masiva afluencia de soldados heridos. En este contexto, Wilson propuso a Sir George Young la habilitación de un hospital infantil, que finalmente quedó instalado en una villa cercana a la universidad.

La cuáquera norteamericana Emily Parker ${ }^{10}$ consideraba que el Hospital Infantil de Murcia había servido para proporcionar, incluso al personal médico local, un concepto nuevo de la atención pediátrica. Las enfermedades más frecuentes tratadas en este hospital fueron sarampión, fiebre tifoidea, meningitis y difteria, aunque las medidas preventivas y terapéuticas instauradas se mostraron eficaces y no hubo ningún caso de contagio nosocomial (Palfreeman, 2014, pp. 176-190; Derby, 2015, pp. 58-92). También se dieron casos de tuberculosis y de tos ferina ${ }^{11}$.

Cuando las tropas de Franco entraron en Murcia en abril de 1939, el personal del hospital hizo saber que su intención era permanecer allí y seguir asistiendo niños bajo el nuevo Régimen, lo que no fue permitido. La nueva dirección (en manos de una falangista) pronto insistió en la clausura del centro, lo que tuvo lugar el 20 de mayo de $1939^{12}$. Los niños -la mayoría muy enfermos- fueron enviados a sus casas; unos pocos de ellos fueron remitidos al colapsado Hospital Provincial, donde fueron atendidos por monjas. Al finalizar la contienda, los cuidados de enfermería en los hospitales, en su mayoría, fueron asumidos de nuevo por las órdenes religiosas tal y como había sido habitual en España (López Vallecillo, 2016, pp. 368-369, 435-436).

Además de los hospitales infantiles de Almería, Murcia y Alicante, la ayuda humanitaria habilitó en la zona republicana una serie de hospitales militares. Así, entre abril y octubre de 1937 se instaló en el edificio de la Escuela Industrial de Alcoi (Alicante) un hospital militar que fue financiado íntegramente gracias a la ayuda desinteresada de miles de ciudadanos 
suecos y noruegos. El llamado «Hospital Sueco-Noruego» llegó a tener 700 camas y estuvo gestionado por una treintenta de voluntarios escandinavos (Beneito Lloris, 2016). El hospital de Alcoi tuvo una estrecha relación con el Hospital Militar Internacional instalado en la vecina localidad de Ontinyent (Valencia). Efectivamente, durante la Guerra Civil funcionó un hospital militar que se habilitó en las dependencias del colegio de La Concepción, que los padres Franciscanos habían regentado en Ontinyent. La elección del emplazamiento no fue casual, dado que Ontinyent estaba situado en la retaguarda republicana y, más importante, estaba bien comunicada gracias a la vía férrea Valencia-Xàtiva-Alcoi. La habilitación del hospital respondió a una iniciativa del Partido Obrero Belga (de tendencia socialista), que contaba con el apoyo de la Internacional Socialista Obrera. Allí trabajaron médicos y enfermeras de diferentes nacionalidades, la mayor parte de las cuales eran judías de origen centroeuropeo que residían en Bélgica. Sorprendentemente, uno de los objetivos de este establecimiento era continuar como hospital civil tras la guerra. Sin embargo, al finalizar el conflicto el hospital acogió a prisioneros heridos y, a finales de 1939, el edificio fue recuperado por los Franciscanos y se volvió a transformar en escuela religiosa (Torró Martínez, 2016, pp. 327-344).

Por otra parte, desde Estados Unidos, el Medical Bureau to Aid the Spanish Democracy impulsó la habilitación y gestión de tres hospitales en la retaguardia de Madrid, donde trabajaron cirujanos como Edward H. Barsky y Parker Goland. En estos hospitales se atendieron gran cantidad de heridos con fracturas craneales $^{13}$. En la primavera de 1937 el Medical Bureau estableció una unidad neuroquirúrgica integrada por tres médicos y cuatro enfermeras en el hospital de las Brigadas Internacionales de Benicàssim, CasteIlón (Casañ, 2006, pp. 161-198), pero en agosto se trasladó a Valencia y se instaló en el Hospital Militar Base (Coni, 2008, pp. 130-131), habilitado en el edificio de la nueva Facultad de Medicina (García Ferrandis, 2015, pp. 277-278).

Finalmente, los británicos contribuyeron a la habilitación de una red de hospitales en la retaguardia republicana, entre los que conviene destacar los denominados «hospitales británicos» de Huete (Cuenca), Uclés (Cuenca) y Valdeganga (Albacete). Habilitados geoestratégicamente, disponían de acceso directo a la vía férrea Madrid-Cuenca y a la carretera MadridValencia, por lo que cubrían la retaguardia de los frentes de Extremadura, Guadalajara y Madrid (Palfreeman, 2016, pp. 305-326).

\section{EL HOSPITAL INFANTIL DE POLOP DE LA MARINA Y LA DESCONOCIDA FIGURA DE MANUEL BLANC RODRÍGUEZ (1899-1971)}

Conviene recordar que tras la ocupación militar de Málaga el 8 de febrero de 1937 se produjo un gran éxodo de población civil hacia las provincias vecinas de Almería y Murcia. El número de refugiados fue tan elevado que gran cantidad de ellos siguieron desplazándose hacia el norte hasta llegar a la provincia de Alicante. Así describía la situación la prensa de la época:

Cuando el éxodo que siguió a la toma de Málaga trajo hasta Alicante a muchos millares de mujeres y niños, la provincia acogió a los refugiados con los mismos brazos abiertos y cordiales con que había ya recibido a los evacuados de Madrid y de otras poblaciones ${ }^{14}$.

En efecto, entre la documentación consultada hemos encontrado numerosas evidencias de la llegada de los refugiados a diferentes localidades alicantinas, que tuvieron que dar acogida a los desplazados. En Crevillent el ayuntamiento habilitó varias colonias y cantinas escolares para los niños refugiados ${ }^{15}$. Asimismo, Francesca Wilson estableció una colonia agrícola en el paraje de "Los molinos» con el fin de acoger a los hijos de los combatientes del Norte. El ayuntamiento se comprometió a acondicionar los accesos a la colonia ${ }^{16}$, que tenía capacidad para 500 niños (Mendlesohn, 2002, p. 96). En Elx también se habilitó una colonia infantil de gestión municipal que, en abril de 1937, acogía a 102 niños $^{17}$. En Benidorm funcionaron al menos dos colonias escolares, una fundada por el ayuntamiento ${ }^{18}$ y otra fundada por Wilson en agosto de 1937 (Mendlesohn, 2002, p. 50) ${ }^{19}$.

Es en este contexto de una provincia de Alicante sometida a una intensa presión demográfica en el que conviene enmarcar la habilitación de un hospital infantil. Efectivamente, los cuáqueros, además de haber fundado los hospitales infantiles de Almería y Murcia, instalaron otro hospital de esas características en la ciudad de Alicante, que abrió sus puertas el 1 de septiembre de $1937^{20}$. La idea inicial era poder atender las necesidades asistenciales de los miles de niños refugiados que había en Alicante tras los acontecimientos de Málaga; sin embargo, dado que la ciudad no disponía de un hospital infantil, la intención 
del FSC era que este centro sanitario continuara funcionando al finalizar la guerra ${ }^{21}$. Sabemos que el centro quedó instalado en una clínica privada de Alicante perfectamente equipada que había sido inaugurada poco antes de la guerra, aunque desconocemos con exactitud dónde se instaló. Siguiendo los antecedentes de Almería y Murcia, los cuáqueros debieron presentar su proyecto de hospital infantil a las autoridades republicanas locales quienes, tras el visto bueno, proporcionaron a los británicos un edificio donde instalar el centro sanitario y destinaron allí a un médico español. En el caso de Alicante, la subsecretaria de Sanidad nombró médico director del «Hospital Inglés de Niños» al "especialista en enfermedades de la infancia» Manuel Blanc Rodríguez ${ }^{22}$.

Nacido en Albacete en 1899, Manuel Blanc fue alistado en el reemplazo de 1920 de Albacete capital después de haber intentado inútilmente conseguir una prórroga por los estudios de medicina que iba a iniciar ese año, participando en diferentes combates en el Rif (Cerro Aguilar, 2007, p. 110). Estudió en la
Universidad de Valladolid, aunque no destacó por ser un estudiante brillante. Cursó varias asignaturas en Madrid y Valencia, suspendiendo curiosamente «enfermedades de la infancia y su clínica» ${ }^{23}$. Tras licenciarse en 1924 realizó diversas estancias en hospitales infantiles de París y Ginebra. Su producción científica fue muy limitada, destacando un artículo en el que defendía los beneficios de la administración intradérmica de la vacuna contra la tos ferina frente a las vías intramuscular y subcutánea (Blanc Rodríguez, 1932, pp. 18-19). Ejerció la pediatría en Madrid entre 1926 y 1936. Con anterioridad a 1949 obtuvo una plaza de puericultor del Estado y fue nombrado jefe provincial en Alicante de los servicios de Higiene Infantil (Bosch Marín, Blanco Otero, Mingo de Benito et al., 1949, p. 6). En 1962 ganaría una plaza de médico del Seguro Obligatorio de Enfermedad en Alicante de la especialidad "Pediatría-Puericultura»²4. En 1952 fundó la Sociedad Médico-Quirúrgica de Alicante (ver imagen 2 ), en la que participó activamente llegando incluso a presidir (Puerta Jiménez, 2015, p. 11, 105). Murió en Madrid en $1971^{25}$.

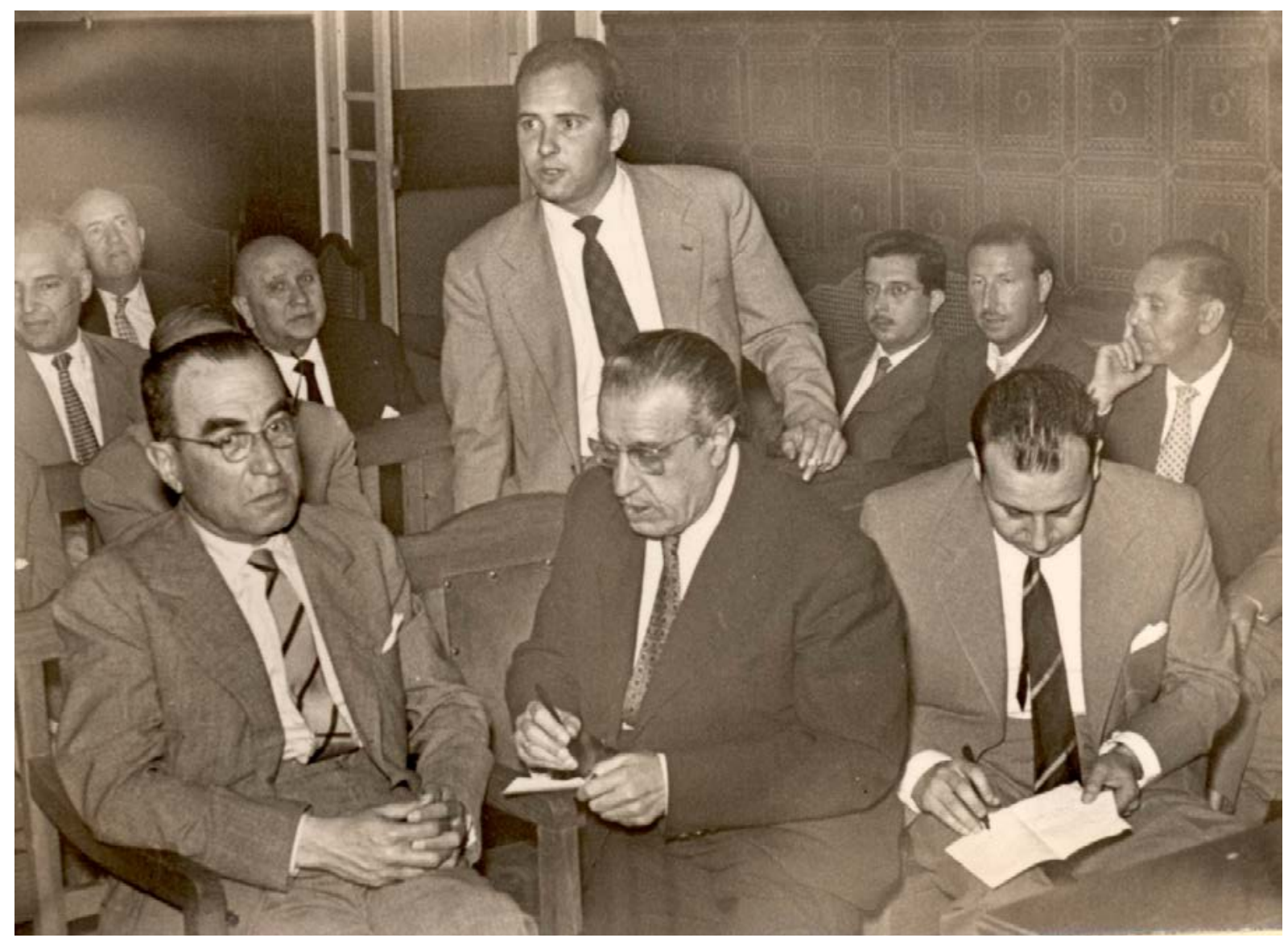

Imagen 2. Manuel Blanc Rodríguez (sentado, centro de la imagen). Fuente: Colegio Oficial de Médicos de Alicante. 
Por motivos que desconocemos -quizá el simple hecho de estar de vacaciones-, el estallido de la Guerra Civil sorprendió a Manuel Blanc en Alicante. En fecha tan temprana como el 22 de julio su hermano Gabriel fue asesinado. Este hecho, junto con sus conocidos antecedentes derechistas y católicos, hizo que buscara y encontrara refugio en una finca propiedad del Dr. Tapia. Manuel Tapia Martínez (nacido en 1895) era doctor en Medicina por la Univeridad de Madrid (1918). Pensionado por la Fundación Rockefeller, entre 1926 y 1927 había estudiado Salud Pública en la Universidad de Harvard (EE.UU). Se especializó en la lucha contra las enfermedades transmisibles, llegando a dirigir el Hospital Nacional de Enfermedades Infecciosas (Madrid). Asimismo, en 1926 ganó una plaza de profesor en la Escuela Nacional de Sanidad, Madrid (Barona y BernabeuMestre, 2008, pp. 96, 97, 132, 133). Seguramente Blanc y Tapia se conocían porque ambos ejercían la medicina en Madrid y veraneaban en Alicante. Al estallar la guerra, Manuel Tapia fue respetado -probablemente por su prestigio-, lo que le permitió proteger a Blanc.

Posteriormente, Manuel Blanc trabajó en el Hospital de la Cruz Roja de Alicante bajo la tutela del Dr. Ruzafa, pero en mayo de 1937 fue expulsado cursándose contra él una orden de detención. Junto con un sacerdote, fue alojado -seguramente escondido- en casa de su benefactor, el Dr. Ruzafa. Asistió al hijo de Jesús Monzón (1910-1973), gobernador civil de Alicante por el PCE, «y aprovechó esta circunstancia para interceder por personas perseguidas por los rojos ${ }^{26}$. Monzón ocupó dicho cargo desde el 18 julio de 1937 hasta el 28 de mayo 1938, cuando fue nombrado gobernador civil de Cuenca (Serrallonga i Urquidi, 2007) ${ }^{27}$.

Nuestra hipótesis es que Blanc «aprovechó esta circunstancia» en favor de su propia seguridad, ya que Monzón, en agradecimiento, habría intercedido ante las autoridades sanitarias republicanas para que Blanc fuera nombrado médico director del «hospital inglés de niños» de Alicante y para que el establecimiento fuera convenientemente abastecido (Martorell, 2000, p. 62). Según esta hipótesis, trabajar para los niños de la República se habría convertido en el seguro de vida de Blanc, lo que, por otra parte, no impidió la incautación de su domicilio y su clínica particular en diciembre de 1937, según consta en su expediente custodiado en el Colegio Oficial de Médicos de Alicante. Asimismo, Blanc fue durante la guerra el responsable de la Gota de Leche de Alicante ${ }^{28}$, que había sido trasladada temporalmente a San Juan y recibía el apoyo financiero del National Joint Committee británico ${ }^{29}$.

La administradora del Hospital Infantil de Alicante era Mary Elmes (1908-2002). Elmes había nacido en Cork (República de Irlanda) y había estudiado literatura moderna española y francesa en el Trinity College de Dublín, y relaciones internacionales en la London School of Economics. Por tanto, Elmes «estaba extremadamente capacitada y hablaba español a la perfección pero no era enfermera» ${ }^{30}$. El conocimiento del castellano y sus habilidades de gestión y organización le hicieron viajar a España de la mano de Sir George Young, aunque como trabajadora contratada por el FSC (Finn, 2017, pp. 37-78). Después de trabajar en el Hospital Infantil de Almería, pasó al de Murcia, posteriormente al de Alicante y finalmente al de Polop de la Marina (a partir de ahora Polop). Tras acabar la guerra de España, pasó al sur de Francia donde gestionó la ayuda humanitaria destinada a los niños españoles y judíos internados en los campos de refugiados del sur de Francia. En 2013, su figura fue reconocida oficialmente por el Yad Vashem, en Israel, con el título de "Justa entre las Naciones" ${ }^{\prime 3}$.

A finales de 1937 el Hospital Infantil de Alicante acogía a unos 30 niños enfermos de fiebre tifoidea, de sarna, de tiña y de tuberculosis; asimismo, todos ellos estaban malnutridos (Mendlesohn, 2002, p. 57). Además de Elmes, el personal del hospital estaba integrado por dos enfermeras británicas. La documentación prueba que una de ellas era Ann Hathaway ${ }^{32}$.

Tras los intensos bombardeos que sufrió Alicante durante la primavera de 1938 , los cuáqueros se dieron cuenta de la necesidad de trasladar el hospital desde Alicante a un emplazamiento más seguro, lo que finalmente tuvo lugar el 11 de junio de $1938^{33}$. El centro sanitario quedó instalado de manera provisional en el Hotel Mediterráneo de San Juan, un pequeño pueblo marinero situado a $10 \mathrm{~km}$ de Alicante. Por aquel entonces el hotel funcionaba como colonia para niños sanos, lo que resultaba incompatible con la acogida de niños enfermos. Otras dificultades añadidas fueron la mala calidad del agua potable y la necesidad de lavar la ropa con agua del mar. Después de barajar distintos emplazamientos, los cuáqueros optaron por trasladar definitivamente el hospital a Polop, un pequeño y tranquilo pueblo rodeado de montañas situado a unos $50 \mathrm{~km}$ de Alicante ${ }^{34}$. Así lo 
explica Dorothy Morris, enfermera neozelandesa destacada en el Hospital Infantil de Murcia: «ellos [los cuáqueros] habían habilitado un magnífico hospital en Alicante pero los bombardeos les obligaron a trasladarlo a un pueblo del interior montañoso, remoto pero muy bello» ${ }^{35}$. El traslado tuvo lugar el 18 de julio de $1938^{36}$ y el hospital quedó instalado en una finca de veraneo que había sido abandonada por su propietario al comienzo de la guerra ${ }^{37}$, un comerciante de pescado que "había huído a un lugar más apropiado para ricos ${ }^{38}$. A nuestro juicio, estas palabras de la enfermera británica Dorothy Litten bien pudieran responder a la hipótesis de que muchos cuáqueros británicos y sus colaboradores se sentían a título personal más cerca de la causa republicana pese a la neutralidad oficial de la agencia humanitaria (Mendlesohn, 2002).

En el marco de nuestra investigación hemos localizado el edificio que albergó el hospital durante la guerra, gracias a las fotografías del archivo personal de Mary Elmes que reconocieron algunos vecinos del pueblo (ver imagen 3). Actualmente, el edificio pertenece a una familia de Madrid que lo utiliza ocasionalmente como segunda residencia ${ }^{39}$. Según el testimonio de unos ellos, en dicho edificio vivían «unas mujeres extranjeras vestidas con una larga bata blanca ${ }^{40}$.

Instalado en Polop, el hospital siguió bajo la administración de Mary Elmes y bajo la dirección médica de Manuel Blanc Rodríguez ${ }^{41}$. Pese a la situación personal del pediatra, todo apunta a que desarrolló su labor médica con gran profesionalidad y dedicación. Las siguientes palabras de Mary Elmes así parecen confirmarlo:

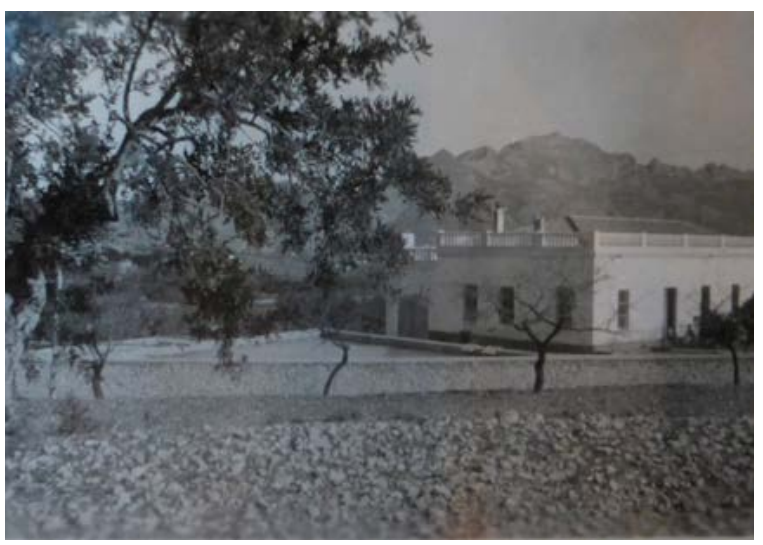

\section{Querida Rose,}

¿Te he hablado alguna vez de Palmira? Era una encantadora niña de 21 meses de edad que fue herida en el bombardeo del mercado de Alicante en 1938 (...). La criatura sufrió una gravísima lesión en una pierna, de tal manera que el pie quedó colgando únicamente sostenido por unas tiras de piel. El cirujano que la atendió en primer momento optó por la amputación del pie. Afortunadamente, el médico de nuestro hospital -que era pediatra- se opuso y trasladó a la niña a nuestro hospital. Allí convaleció con la pierna inmovilizada durante tres meses. Tras este período fue capaz de levantarse y empezar a dar pequeños pasos con normalidad. Fue un triunfo del Doctor Blanc y de las enfermeras inglesas, quienes deberían recibir un reconocimiento por su dedicación y paciencia $(\ldots)^{42}$.

En su carta Mary Elmes se refiere al brutal bombardeo que sufrió el mercado central de Alicante el 25 de mayo de 1938 y que ocasionó más de 300 muertos, la mayoría mujeres y niños. En el archivo personal de Mary Elmes existe una fotografía donde se observa a Palmira con su pierna derecha vendada, una vez evacuada al Hospital Infantil de Polop. La fotografía (ver imagen 4) está fechada el mismo día del ataque, lo que reforzaría la idea un diligente y resolutivo Manuel Blanc.

Blanc recogía a determinados niños procedentes de los distintos refugios de la costa de Alicante y los llevaba él mismo en coche al hospital de Polop dos veces a la semana. El resto de días o en caso de urgencia existía la posibilidad de llamar al médico titular de la localidad $^{43}$. En general, los niños trasladados a Polop estaban muy pálidos y, día a día, mejoraban gracias al sol y al aire puro de las montañas. Todos ellos estaban demacrados y envejecidos a causa de la malnutrición.

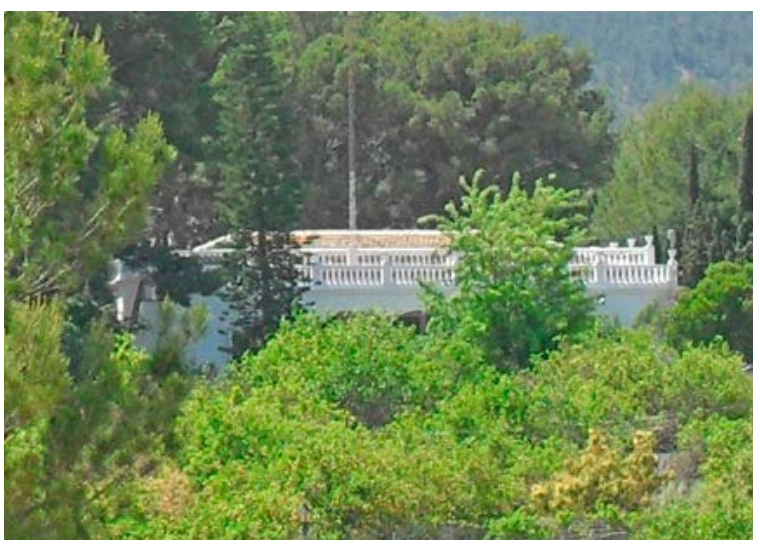

Imagen 3. Edificio en Polop de la Marina en el que se instaló el hospital infantil: aspecto durante la guerra y en la actualidad. Fuente: archivo de Mary Elmes (izquierda) y archivo de Bernard Wilson (derecha). 


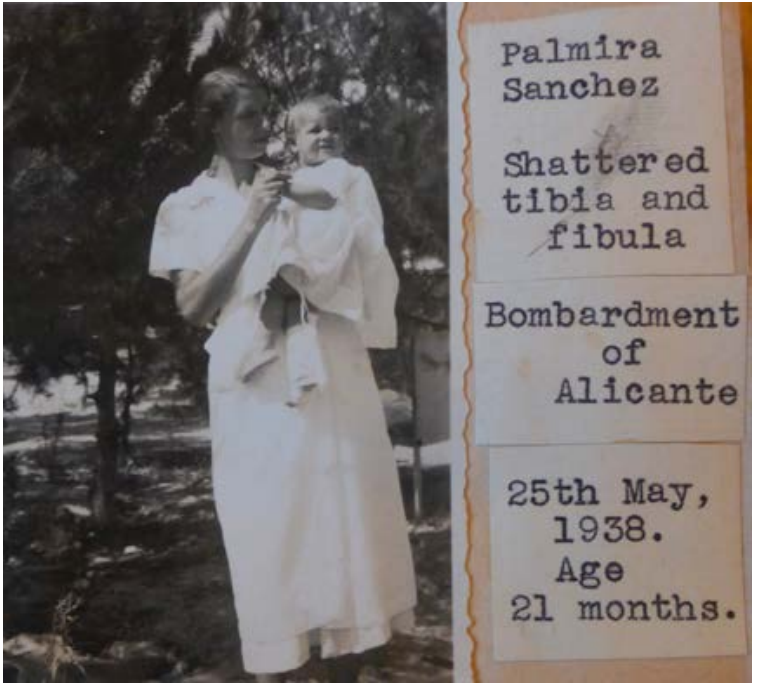

Imagen 4. Mary Elmes sosteniendo en brazos a la pequeña Palmira herida. Fuente: archivo personal de Mary Elmes.

Aunque algunos niños llegaban en unas condiciones médicas de extrema gravedad y morían, la mayoría simplemente necesitaban una buena alimentación y un estilo de vida de acuerdo a su edad.

El tipo de paciente que Blanc trasladaba a Polop era reflejo de las penurias que se sufrían en la retaguardia: dos de los niños habían sido víctimas de los bombardeos de Alicante de final de mayo. Otros estaban afectados por enfermedades carenciales (ver imagen 5). En un caso se trataba de un niño llamado Rafaelito que había sido encontrado vagando solo por las calles de Alicante. Era tan pequeño que no sabía ni su nombre y llegó al hospital en muy malas condiciones: afectado de escorbuto, presentaba una malnutrición tan extrema que le impedía si quiera mantenerse de pie. Cuando mejoró, pasó a comer en la mesa con el resto de niños pero, en una clara evidencia de falta de hábitos de alimentación, no sabía utilizar la cuchara.

Los casos de niños deambulando solos por las carreteras y las calles, que Dorothy Litten asociaba a la huida desesperada y precipitada de grandes masas de población ${ }^{44}$, eran frecuentes. Estos casos se dieron en la trágica huida de Málaga, en la Retirada (Kershner, 2011, pp. 88-89) y en la evacuación de las colonias infantiles de Puigcerdà a Francia (Finestres, 2012, p. 12), entre otras.

Otro tipo de enfermos cuyo manejo resultaba especialmente díficil eran aquellos afectados por enfermedades infecciosas. En una ocasión Manuel Blanc ordenó el ingreso de un niño llamado Ángel. Ante un posible diagnóstico de difteria, los respon-

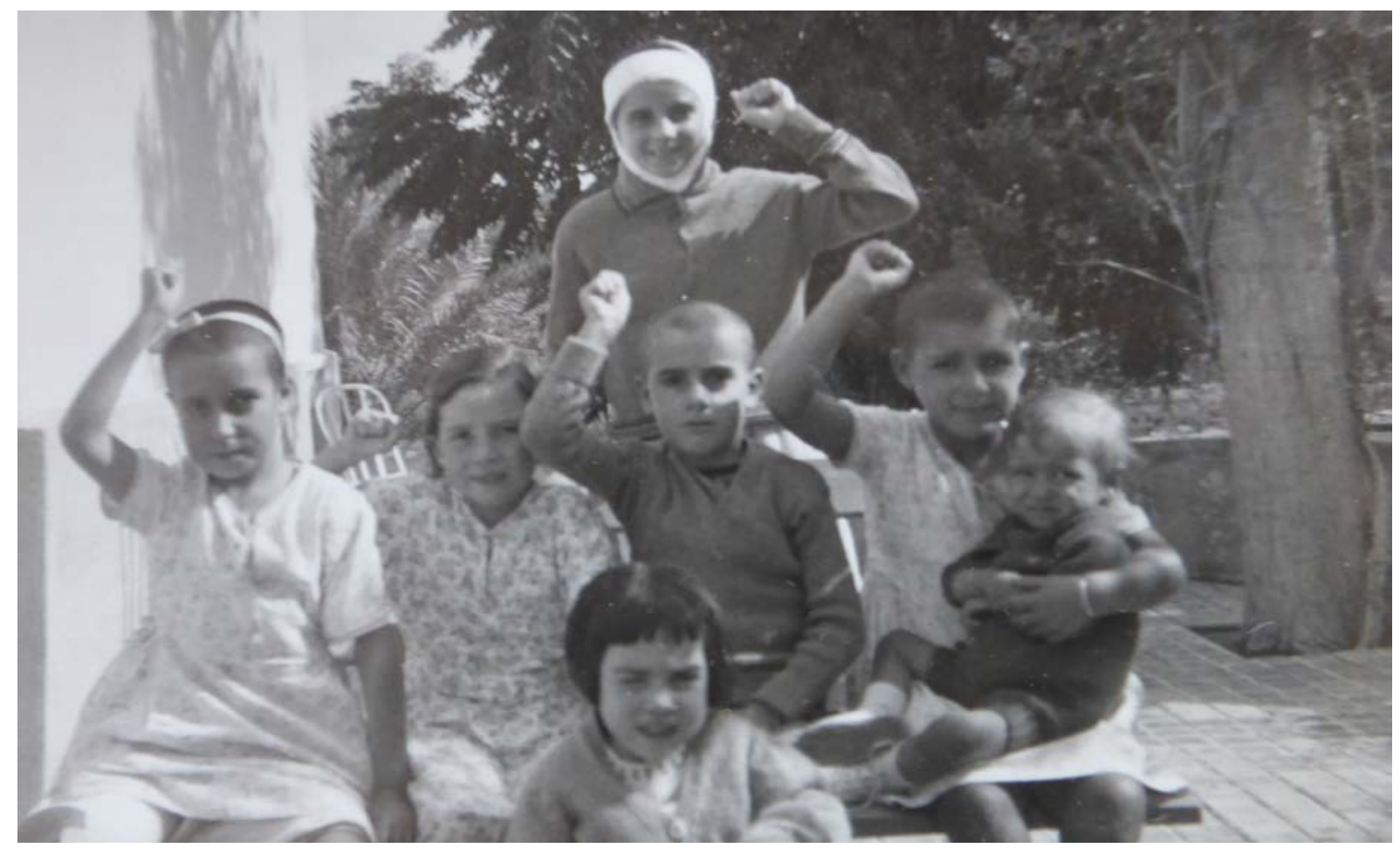

Imagen 5. Niños acogidos en el hospital de Polop de la Marina. Fuente: archivo personal de Mary Elmes. 
sables tomaron medidas para evitar el contagio de unos 30 niños, por lo que Ángel y los dos niños que dormían a su lado fueron aislados en una pequeña habitación. El aislamiento no fue suficiente y se declararon otros casos, aunque finalmente la infección fue controlada gracias a la aplicación de suero antidiftérico.

En cuanto al personal español, además del médico, el hospital contaba con seis auxiliares de enfermería, un portero, un cocinero y una o dos personas encargadas de la limpieza ${ }^{45}$.

Por su parte, el personal de enfermería británico durante el tiempo que el hospital estuvo en Polop estuvo integrado por Rachel Marshall, Dorothy Litten, Marjorie Kent e Irene Callon ${ }^{46}$. En realidad no eran cuáqueras, sino personal sanitario cualificado contratado por el FSC para trabajar en los hospitales de Almería, Murcia y Alicante (Mendlesohn, 2002, p. 192). Cabe señalar que no todas las enfermeras coincidieron en el tiempo, ya que el FSC tenía establecido un sistema de rotación y uno de descanso en forma de vacaciones pagadas. Así, el personal del hospital de Polop fue destinado eventualmente a los hospitales infantiles de Murcia y Almería, y viceversa. El sistema de rotación no descartaba, incluso, el cambio de bando en conflicto. Por ejemplo, en mayo de 1938 el cuáquero californiano Clyde Roberts, que había trabajado en la administración del hospital de Murcia, sustituyó a su compañero Earl Smith, quien venía desempeñando su función en la España franquista ${ }^{47}$.

Las enfermeras contratadas por el FSC recibían un salario que normalmente se pagaba cuando las trabajadoras regresaban al Reino Unido. El salario consistía en 10 chelines a la semana durante los primeros tres meses y una libra semanal posteriormente ${ }^{48}$. Además, estaba estipulado que cada trabajador destacado por su labor en España tuviera un mes de vacaciones por cada seis de meses de trabajo, remunerado con tres libras semanales ${ }^{49}$. Por ejemplo, en noviembre de 1938 Mary Elmes se encontraba de vacaciones, período que aprovechó para desplazarse a Madrid al objeto de comprobar la situación de la población civil. En aquellos momentos Irene Callon asumió la administración del hospital de Polop mientras que Rachel Marshall desempeñó la labor enfermera, teniendo a su cargo a «33 o 34 niños, un número record en Polop $»^{50}$. Las enfermeras que durante su período vacacional no regresaban al Reino Unido tenían derecho a solicitar un permiso cuando, final- mente, regresaban a casa. Los responsables cuáqueros pensaban que «la razón de que dispongan de permisos vacacionales es permitirles descansar y recuperarse de la presión del trabajo en España $»^{51}$. El hecho de tener un salario y poder disfrutar de vacaciones pagadas no debería de hacernos dudar de la auténtica vocación, dedicación y profesionalidad del personal, convirtiéndolas en una suerte de «mercenarias del humanitarismo». Así, por ejemplo, Mary Elmes disfrutó de un permiso vacacional mucho más corto del que le correspondía y, además, pagó de su bolsillo los gastos que se derivaron ${ }^{52}$. Conviene tener en cuenta, además, que el trabajo se desarrollaba en unas condiciones muy duras, tanto físicas (exceso de horas de trabajo, hambre) como psicológicas (bombardeos, gran responsabilidad, impotencia y rabia al ver morir a niños inocentes). Precisamente el objetivo del sistema de rotación y de vacaciones pagadas ideado por los dirigintes cuáqueros era minimizar el impacto psicológico en sus trabajadores. Nos consta que este procedimiento fue efectivo con alguna excepción, como el caso de Ann Hathaway. Hathaway empezó a trabajar como enfermera en el Hospital Infantil de Alicante nada más inaugurarse (septiembre de 1937) y renunció a sus vacaciones pese a la insistencia de los responsables cuáqueros. En marzo de 1938 presentaba un estado de agotamiento tal que fue considerada «no apta para el trabajo, tanto desde el punto de vista físico como emocional». Por este motivo, le obligaron a descansar unas semanas en un pueblo de Murcia, pero al cabo de poco tiempo manifestó su deseo de reincorporarse. Fue entonces cuando se decidió trasladarla al hospital de Almería; sin embargo, los responsables cuáqueros consideraron finalmente que Hathway «no disponía en absoluto de las habilidades emocionales que exige un trabajo de estas características ${ }^{53}$. Finalmente, Ann Hathaway fue repatriada al Reino Unido.

La enfermera británica Dorothy Litten ${ }^{54}$ había trabajado anteriormente como matrona en los hospitales que los cuáqueros tenían destacados en el Líbano y en Siria. En su camino hacia Polop, Litten aterrizó en el aeropuerto de Barcelona en medio de un bombardeo y pudo observar una columna de polvo y piedras que se elevaban hacia el cielo: era el verano de 1938 (Jackson, 2010, p. 150). Dorothy Litten llegó a Alicante dos días antes del traslado del hospital a Polop. A su llegada al aeropuerto de Alicante, describe un "calor abrasador» y un control de aduanas «muy estricto». Al pasar por la ciudad camino de 
Polop, Litten se dio cuenta de que Alicante había sido bombardeada aquella misma mañana, viendo un barco medio hundido en el puerto y varios edificios en llamas. En el momento de la llegada de Dorothy Litten a Polop, el hospital acogía a 23 niños y la capacidad total del hospital rondaba las 40 camas $^{55}$. Litten llegó a Polop para relevar a Marjorie Kent, que se encontraba de vacaciones, haciéndose cargo de la dirección de enfermería. Durante su labor en el hospital, estuvo auxiliada por seis mujeres españolas, todas ellas refugiadas y sin una formación básica en enfermería. Esto obligó a Litten a supervisarlas constantemente, pese a la dificultad añadida de la diferencia de idioma. Determinados casos clínicos llamaban especialmente la atención de Dorothy Litten:

El último ingreso se trata de un niño que sufre escorbuto y que tiene una enorme necesidad de afecto. Cada vez que me acerco a él, señala con sus brazos el lugar donde quiere que le traslade. La impresión que tengo es que está buscando algo, seguramente acaba de perder su familia (...). No le gusta estar en el interior del hospital, por lo que hemos instalado su cuna en el jardín para que no se queje ${ }^{56}$.

Efectivamente, el suave clima mediterráneo permitía colocar ocasionalmente las cunas y las camas en las terrazas exteriores del hospital (ver imágenes 6 y 7$)$.

La determinación y la entrega de Litten con los niños que atendía se hace patente al afirmar «son niños adorables, mucho más encantandores y menos tímidos que los niños ingleses o los sirios». Dorothy Litten redactó su informe una calurosa noche de agosto de

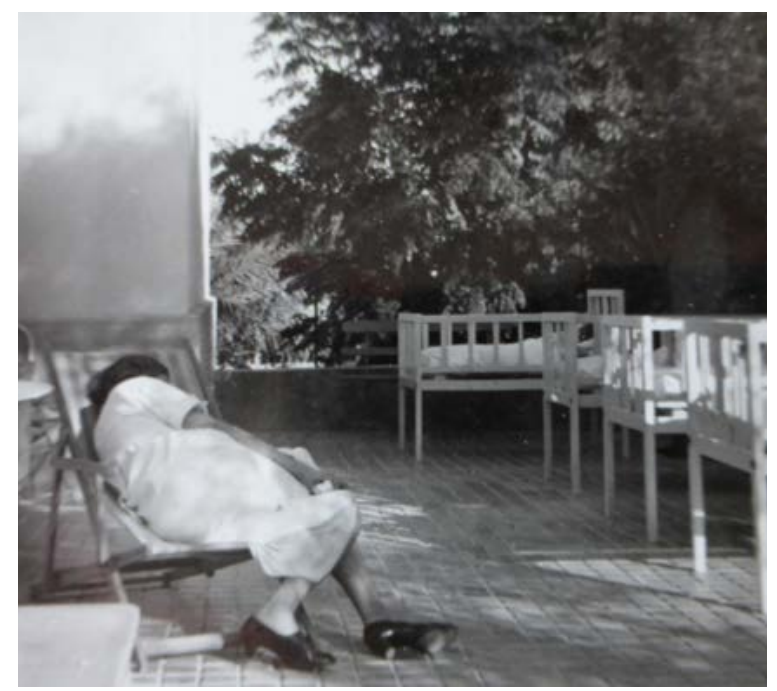

1938 cuando los niños dormían tranquilamente alejados de las bombas: «Estamos profundamente agradecidas por este oasis de paz. Este es el refugio que los Friends han hecho posible para niños que han perdido la seguridad de su propio hogar ${ }^{57}$. Pese a la tranquilidad del lugar, las enfermeras nunca olvidaron la guerra, ya que desde Polop cuando soplaba viento de levante se oían los bombardeos de las ciudades costeras; incluso en alguna ocasión divisaron los aviones de regreso a su base en Mallorca. Tras dos meses de trabajo en Polop, Dorothy Litten fue destinada al Hospital Infantil de Murcia y fue sustituida por Rachael Marshall.

Polop también acogía gran número de mujeres y niños que habían sido evacuados de Madrid $^{58}$. Los muchachos más mayores ayudaban en las tareas del jardín y del campo plantando hortalizas y regando, ya que la finca donde estaba instalado el hospital disponía de dos balsas de riego. Cuando estaban llenas servían para lavar a los niños que estaban bien de salud antes de irse a dormir. Las tierras de la finca se habían dividido entre cinco agricultores que las cultivaban para ellos mismos ${ }^{59}$, aunque recibían la ayuda de los niños más mayores.

Dado que muchos niños mejoraban simplemente con una buena alimentación, se hizo necesaria la educación de los pequeños. Así, sabemos que los niños recibían, además de asistencia médica, ropa y juguetes, una instrucción elemental a cargo de dos maestros españoles (Mendlesohn, 2002, p. 57), como se puede apreciar en la imagen 8.

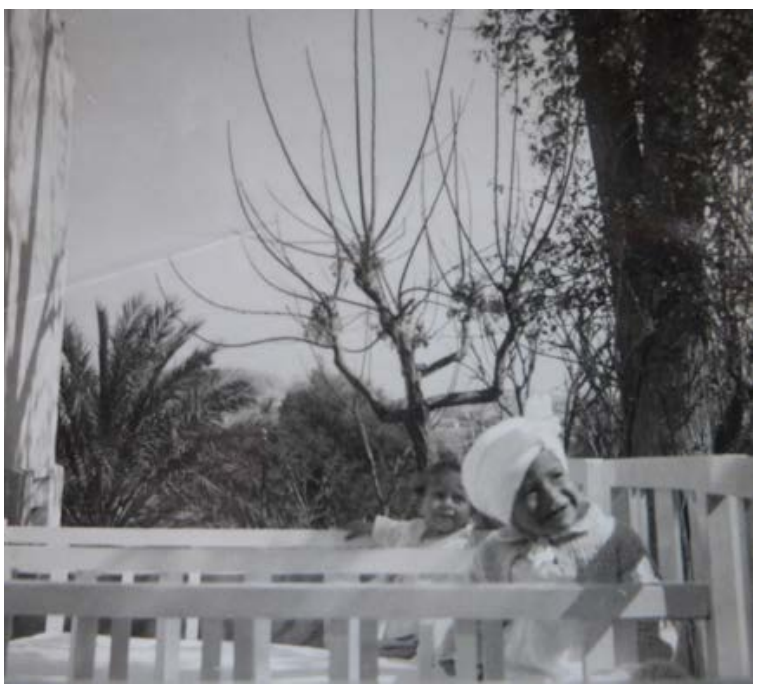

Imágenes 6 y 7. Cunas en el exterior del hospital. Fuente: archivo personal de Mary Elmes. 


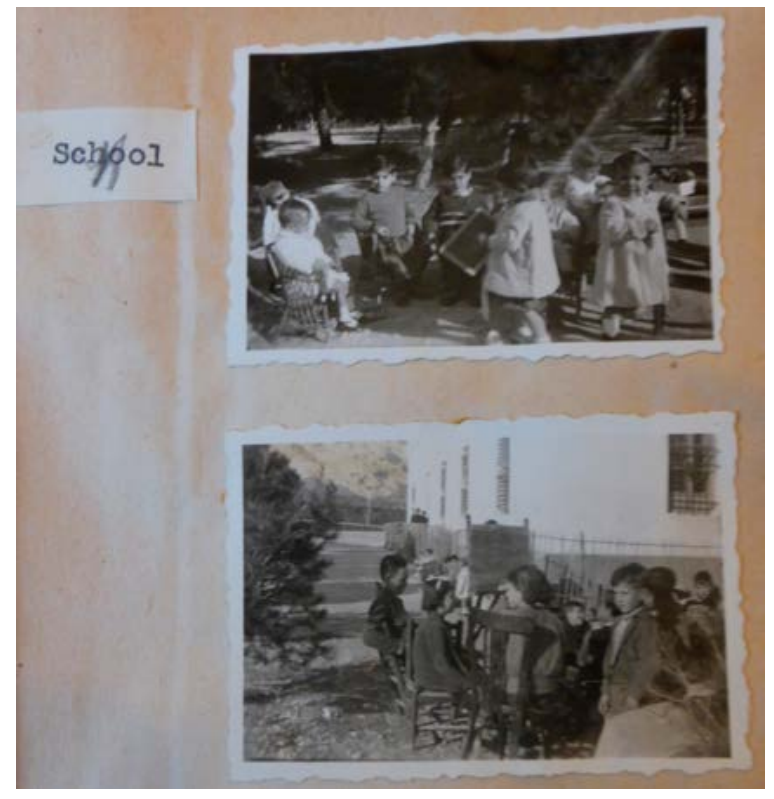

Imagen 8. Escuela improvisada en el hospital de Polop. Fuente: archivo personal de Mary Elmes.

Al igual que el resto de la retaguardia republicana, el hospital de Polop padeció la carestía de alimentos. En agosto de 1938 el hospital presentaba serios problemas de abastecimiento: varias toneladas de trigo enviadas desde América se habían extraviado y los suministros médicos solicitados en mayo todavía no habían llegado ${ }^{60}$. Una mañana Dorothy Litten fue al mercado de Polop con Juan, un niño refugiado de Málaga. Llevaban un burro cuyas alforjas pretendían llenar con alimentos para los niños del hospital. Sin embargo, cuando llegaron al mercado tan solo había dos puestos que vendían unas pocas frutas y verduras. Cuando regresaron a la semana siguiente, ya no había nada disponible porque el Gobierno había fijado los precios tan bajos que nadie quería vender nada. Cuando Litten abandonó España en enero de 1939 en el mercado de Polop solo quedaban espinacas $y$, de vez en cuando, unas pocas naranjas ${ }^{61}$. Esta situación de desabastecimiento de la población civil también fue descrita por Dorothy Morris: «estamos sufriendo un bloqueo y nos morimos de hambre; nosotros (los cuáqueros) somos los únicos que disponemos de leche y comestibles (...) $»^{62}$.

Finalmente, el Hospital Infantil de Polop fue clausurado el 5 de mayo de $1939^{63}$ en un contexto general de animadversión del nuevo Régimen hacia los cuáqueros y sus colaboradores. Tal y como recuerda Dorothy Morris, jefa de Enfermería del Hospital Infantil de Murcia: "Los nacionalistas (Franco) se han incau- tado de toda la comida y los suministros (a pesar de las promesas de hacer lo contrario) y han cerrado las cantinas y los hospitales de Murcia y Alicante ${ }^{64}$. Morris, amiga de Mary Elmes, con quien trabajaría posteriormente en los campos del sur de Francia, había visitado en varias ocasiones el hospital de Polop (Derby, 2015 pp. 74, 85, 86). El testimonio de Morris coincide con Jim Fyrth: «En junio de 1939 el FSC comunicó que toda evidencia del trabajo realizado por los cuáqueros en España había desaparecido prácticamente de la noche a la mañana» (citado en Palfreeman, 2014, p. 190).

Respecto de la suerte de los niños que se encontraban ingresados, sabemos por un oficio del alcalde de Alicante, dirigido al de la localidad murciana de Cieza, que:

El llamado Hospital Inglés (...) fué trasladado de esta ciudad [Alicante] al pueblo de Polop, de esta provincia, y al ser liberado dicho pueblo, los niños del citado hospital fueron diseminados por la provincia de Valencia ${ }^{65}$.

Sin embargo, los planes de Sir George Young para los hospitales infantiles de Murcia y Polop eran bien diferentes. Tras el final de la guerra, la vuelta a casa de los refugiados malagueños desde Murcia y Alicante, y la recuperación por parte de sus propietarios de los edificios que habían albergado los centros sanitarios, hizo que el diplomático británico ofreciera a los cuáqueros británicos su mansión en Torremolinos para acoger los equipos y los pacientes de ambos hospitales, asumiendo asimismo los gastos que se derivaran ${ }^{66}$.

\section{CONCLUSIONES}

Tanto la motivación del Friends Service Council para habilitar un hospital infantil como su ubicación no fueron casuales, más bien respondieron a criterios geoestratégicos. En primer lugar, fue instalado en la ciudad de Alicante como respuesta sanitaria a la crisis humanitaria que se produjo tras la entrada de las tropas italianas en Málaga en febrero de 1937, que provocó un éxodo masivo y dramático de la población civil, fundamentalmente ancianos, mujeres y niños. La inestabilidad del frente de Motril (Granada) -que parecía anunciar la inminente caída de Almería- y el insistente acoso bélico por mar y aire sobre esta población hizo que muchos refugiados andaluces continuaran huyendo, en primer lugar a Murcia y, posteriormente, a Alicante. Los bombardeos a que se vió 
sometida esta ciudad a partir de la primavera de 1938 obligó a los responsables del hospital a trasladarlo a un lugar más seguro, siendo Polop de la Marina el lugar adecuado.

Los niños ingresados en el Hospital Infantil de Polop fueron fiel reflejo de las penurias por la que atravesó la zona republicana una vez se consolidó y se prolongó un escenario de guerra civil: traumatismos por los bombardeos, enfermedades carenciales debido a la malnutrición y enfermedades infecciosas como la difteria. Generalmente, tras un tratamiento adecuado y una alimentación saludable los pacientes se recuperaban rápidamente y pasaban el día jugando por los campos de la finca, e incluso tuvieron la posibilidad de recibir una instrucción básica tal y como evidencian algunas fotografías del archivo personal de Mary Elmes. Así pues, se puede apreciar como en este hospital infantil se conjugaron las tres facetas fundamentales del humanitarismo en tiempos de guerra: la asistencia médica, la provisión de alimentos y la educación.

El Hospital Infantil de Polop compartió una peculiaridad con otros centros sanitarios habilitados por la ayuda transnacional (como el de Murcia y el de Ontinyent): su rápida clausura al terminar la guerra pese a la intención de sus responsables de su continuidad al finalizar la guerra. A nuestro juicio, convendría buscar motivos de carácter político y religioso, es decir, argumentos ideológicos a la hora de determinar su cierre; para el caso concreto del Hospital Infantil de Polop, conviene destacar que se había fundado y gestionado en una zona republicana de la mano de una organización extranjera protestante que, además, procedía de un país con una trayectoria democrática claramente consolidada. En un contexto de reconversión obligatoria de la población al catolicismo, la actividad pública de una confesión no católica como los cuáqueros resultaba inadmisible para un régimen que pretendía implantar una sola fe religiosa, con el fin de promover el pensamiento único sobre la base de eliminar cualquier discrepancia política o ideológica. Asimismo, existen indicios que permitirían entrever una cierta inclinación -en el contexto de la neutralidad oficial- de muchos cuáqueros y sus trabajadores hacia la causa republicana y las ideas socialistas. Por todo ello, las autoridades franquistas cerraron los hospitales de los cuáqueros a medida que las tropas iban entrando en las diferentes ciudades porque consideraban que los niños que allí se atendían eran "rojos". En los primeros compases del nuevo Régimen, la asistencia sanitaria infantil fue utilizada como correa de transmisión de los valores del nacionalcatolicismo, para lo que contaron con la complicidad de Falange (Auxilio Social) y de la Iglesia católica. Así, los hospitales infantiles habilitados por la ayuda transnacional fueron progresivamente clausurados, y los niños que allí se encontraban ingresados fueron remitidos a los viejos hospitales preexistentes antes de la guerra, bajo la tutela de la monjas, con el objetivo de adoctrinarlos a la vez que convalecían. Por tanto, todo parece indicar que la asistencia social (distribución de alimentos y de ropa) y la asistencia sanitaria infantil formaban parte de una estrategia para la legitimación del nuevo Régimen y que ambas se pusieron al servicio del nuevo Estado. La estrategia de consolidación del Régimen mediante la asistencia sanitaria continuaría tras la guerra, siendo el tifus exantemático paradigma de esta realidad (Jiménez Lucena, 1994, p. 188).

Por lo que respecta al director médico del Hospital Infantil de Polop, nuestra investigación ha rescatado la identidad, figura y trayectoria de Manuel Blanc Rodríguez. Pediatra domiciliado en Madrid, el estallido de la guerra le sorprendió en Alicante. Debido a sus antecedentes políticos y religiosos corrió toda suerte de infortunios que le debieron hacer temer por su vida. En un primer momento contó con la ayuda de su colega Manuel Tapia Martínez, uno de los salubristas más prestigiosos de los años 20 y 30 . Posteriormente, su amistad con Jesús Monzón -miembro del PCE y gobernador civil de Alicante-impulsó su nombramiento como director del «hospital inglés de niños», cargo que le alejó de la escena política y, eventualmente, le puso a salvo. No hemos evidenciado, así pues, una motivación humanitaria en la decisión de Blanc, lo que no entraría en contradicción con su profesionalidad, entrega y dedicación a sus pequeños pacientes.

\section{AGRADECIMIENTOS}

Este trabajo se enmarca en el proyecto de investigación financiado por la Generalitat Valenciana “Ayuda sanitaria internacional durante la Guerra Civil española (1936-1939): el caso de los cuáqueros británicos" (GV/2015/013), y en el proyecto financiado por el Ministerio de Economía y Competitividad (MINECO) "Acciones de socorro y tecnologías médicas en emergencias humanitarias (1850-1950): agencias, agendas, espacios y representaciones" (HAR2015-67723-P). 
Asimismo agradecemos a las siguientes personas la ayuda prestada para la elaboración de este trabajo: a Caroline y Patrick, hijos de Mary Elmes, por permitirnos el acceso y la reproducción del archivo fotográfico de su madre; a Bernard Wilson, por facilitarnos documentos custodiados en la Friends House Library de Londres; a Linda Palfreeman, por propor-

\section{NOTAS}

1. Una primera versión fue presentada en Ginebra el día 28 octubre de 2016 en el simposio internacional Warriors without weapons. Humanitarian action in the Spanish Civil War and the Republican exile, organizado con ocasión del $80^{\circ}$ aniversario del comienzo de la contienda. En el XVII Congreso de la Sociedad Española de Historia de la Medicina celebrado en Sant Feliu de Guíxols (Girona) los días 15-18 de junio de 2017, los autores presentaron una comunicación que planteaba las dificultades metodológicas para abordar el hospital objeto de este estudio desde una perspectiva de género. La traducción de las citas literales del inglés al castellano ha corrido a cargo de Xavier García Ferrandis.

2. "Rapport sur la mission sanitaire en Espagne , 1937", Revista de Sanidad e Higiene Pública, 12 (5), pp. 297-332.

3. Para conocer en profundidad la figura de Alfred Jacob y la labor del FSC en Barcelona, consúltese Mendlesohn, 2002, pp. 23-46.

4. Según las diferentes fuentes consultadas el bombardeo de Gernika ocasiónó entre 120 y 300 víctimas mortales. El crimen cometido en la carretera Málaga-Almería se saldó con entre 3.000 y 5.000 muertos.

5. Estadística de Refugiats als pobles de la Cerdanya. 1937. Refugiats 1936-1937, 9.3. Arxiu Comarcal de la Cerdanya (Puigcerdà, Girona).

Para saber más sobre la asistencia a los refugiados infantiles en Puigcerdà impulsada por la ayuda humanitaria transnacional, consúltese Finestres, 2012.

6. "Diario del Refugiado", Sembrador. Órgano de la Juventudes Libertarias, Año 1, número 37, 4 abril 1937. Consultado en el Arxiu Comarcal de la Cerdanya (Puigcerdà, Girona) en octubre de 2015.

7. État des enfants qui doivent partir pour la colonie. 25 septiembre 1940. Records Relating to Humanitarian Work in France, 1933-1950. Series I, Perpignan Office, Sub-series: Programs, box 1, folder 2. Archivo del American Friends Service Committee (AFSC, Filadelfia, EE.UU).

8. Para conocer los motivos por los que el AFSC disponía de más fondos y recursos que el FSC, consúltese Mendlesohn, 2002 , pp. 12, 13. Para conocer a fondo la figura de Esther Farquhar, consúltese Mendlesohn, 2002, pp. 52-62.

9. Para conocer en profundidad la figura y la trayectoria de Francesca Wilson consúltese Wilson, 1944 y Mendlesohn, 2002, pp. 47-50. cionarnos el manuscrito Six months in Southern Spain y documentos del archivo de la familia Young (Londres); a Mack Morum, por facilitarnos documentación custodiada en la National Library of New Zealand; al Colegio Oficial de Médicos de Alicante; $y$ a los vecinos de Polop de la Marina, que nos han ofrecido sus testimonios personales.

10. Para obtener más información sobre Emily Parker, consúltese Mendlesohn, 2002, pp. 51-70 y pp. 150-158.

11. From a letter from Sylvia Pitt, Murcia, dated 27 July 1938. Friends Service Council, box FSC/R/SP/3/1, Murcia. Friends House Library (Londres).

12. Archivo personal de Mary Elmes.

13. From a Hospital in Spain. American Nurses Write (1937), Nueva York, Medical Bureau to Aid Spanish Democracy. Consultado en el Pavelló de la República (Barcelona) en octubre de 2015 .

14. De la Serena, Antonio, "Alicante. Como viven los evacuados de Madrid", Mundo Gráfico, 26 mayo 1937.

15. Actas del Consejo Municipal de Crevillente. 25 mayo 1937. 13 enero 1938. Arxiu Municipal de Crevillent (Alicante).

16. Actas del Consejo Municipal de Crevillente. 30 diciembre 1937. Arxiu Municipal de Crevillent (Alicante). Para más información sobre esta colonia agrícola, consúltese Wilson, 1944, pp. 193-196.

17. Oficio del Ministerio de Instrucción Pública y Bellas Artes referente a la Colonia Pro-Infancia de esta Ciudad.1937. D 187/2, Correspondència 1937, legajo 153, núm. 19. Arxiu Històric Municipal d'Elx.

18. Actas del Ayuntamiento de Benidorm. 13 septiembre 1936. Arxiu Municipal de Benidorm.

19. Para más información sobre la colonia agrícola de Crevillent y la marítima de Benidorm, consúltese Wilson, 1944, pp. 193-196 y pp. $200-206$.

20. Archivo personal de Mary Elmes.

21. Davies, Dorothy, Six months in Southern Spain. [s.f]. Box D-2: AC/1. Marx Memorial Library (Londres).

22. "Nombramiento merecido", El Luchador, 1 noviembre 1938.

23. Expediente de Manuel Blanc y Rodríguez. LEG-646-13. Archivo histórico de la Universidad de Valladolid.

24. Boletín Oficial del Estado, núm 93, p. 5215.

25. $A B C, 16$ enero 1972, p. 110.

26. Expediente de Manuel Blanc Rodríguez. Archivo del Colegio Oficial de Médicos de Alicante.

27. Tras el final de la Guerra Civil, Monzón pasó a Francia donde organizó la resistencia de los republicanos españoles contra los nazis. Al finalizar la Guerra Mundial fue uno de 
los principales promotores de la Operación Reconquista. Para conocer más sobre la figura y trayectoria de Jesús Monzón puede consultarse Martorell, 2000 y el documental "Jesús Monzón, el lider olvidado por la historia" (2011), disponible en https://vimeo.com/25125706.

28. Las gotas de leches fueron la fórmula institucional de lucha contra la mortalidad infantil que, procedente de Francia, se generalizó en España a principios del siglo XX. A modo de consultorio, se informaba a las madres sobre la forma de alimentar a sus hijos, promoviendo la lactancia materna y, llegado el caso, facilitando la lactancia artificial. Para conocer más sobre las gotas de leche en España puede consultarse Rodríguez-Ocaña, 1996, pp. 149-169.

Para conocer más sobre la Gota de Leche de Alicante, consúltese Perdiguero-Gil y Bernabeu-Mestre, 1999, pp. 291310; Perdiguero-Gil y Bernabeu-Mestre, 2005, pp. 347-359.

29. Gota de Leche. Friends Service Council, box FSC/R/SP/3/3, Valencia: letters from. Friends House Library, Londres (a partir de ahora $\mathrm{FHL}$ ).

30. Davies, Dorothy, Six months in Southern Spain. 1938. Box D-2: AC/1. Marx Memorial Library (Londres).

31. Sobre la figura de Mary Elmes puede consultarse, además de los trabajos enumerados anteriormente sobre los cuáqueros, la contribución de Wilson, 2012, y las monografías de Butler, 2017, y Finn, 2017, alentadas por el reconocimiento internacional, en especial por el Estado de Israel. En su conjunto, se centran en la labor desarrollada por Elmes en el sur de Francia, considerando su presencia en la Guerra Civil española como un mero antecedente.

32. Letter to Edith Pye, dated March 20th 1938. Friends Service Council, box FSC/R/SP/3/1, Murcia, FHL.

33. Archivo personal de Mary Elmes.

34. Copy of the report received from Dorothy Litten dated August 2nd, 1938. Friends Service Council, box FSC/R/ $\mathrm{SP} / 3 / 3$, Valencia: letters from. FHL.

35. Letters from Dorothy Morris. 5 enero 1939. MS-Papers-9144. Alexander Turnbull Library, National Library of New Zealand, Wellington (Nueva Zelanda).

36. Archivo personal de Mary Elmes.

37. Davies, Dorothy, Six months in Southern Spain. 1938. Box D-2: AC/1. Marx Memorial Library (Londres).

38. Copy of the report received from Dorothy Litten dated August 2nd, 1938. Friends Service Council, box FSC/R/ $\mathrm{SP} / 3 / 3$, Valencia: letters from. FHL.

39. Información facilitada por Bernard Wilson.

40. Testimonio oral de la Sra. Gadea. Entrevista realizada el 20 de mayo de 2012 en Polop de la Marina.

41. Friends Service Council, box FSC/R/SP/3/3, Valencia: letters from. FHL.

42. Carta de Mary Elmes, 1997. Inédita. Proporcionada a los autores por Bernard Wilson.

43. Friends Service Council, box FSC/R/SP/3/3, Valencia: letters from. FHL.
44. Copy of the report received from Dorothy Litten dated August 2nd, 1938. Friends Service Council, box FSC/R/ $\mathrm{SP} / 3 / 3$, Valencia: letters from. FHL.

45. Friends Service Council, box FSC/R/SP/3/3, Valencia: letters from. FHL.

46. Como se puede observar, a excepción del médico, todo el personal sanitario destinado en el Hospital Infantil de Polop era femenino. Para conocer desde una perspectiva de género la labor humanitaria emprendida por las mujeres, enfermeras o no, durante la Guerra Civil española, puede consultarse Martín Moruno, 2009, 2010, 2017. Con respecto a la trayectoria de las enfermeras británicas que allí trabajaron, véase una primera aproximación en García Ferrandis y Martínez-Vidal, 2017.

47. Letter to Esther Farquhar from Dorothy Thomson, dated May 26th 1938. Friends Service Council, box FSC/R/SP/3/1, Murcia. FHL. Información complementada con datos consultados en Mendlesohn, 2002, pp. 61 y 84.

48. Letter to Esther Farquhar. Friends Service Council, box $\mathrm{FSC} / \mathrm{R} / \mathrm{SP} / 3 / 1$, Murcia. FHL.

49. Letter from Ruth Cope to Emily Hughes, dated January 24th 1939. Friends Service Council, box FSC/R/SP/3/1, Murcia. FHL.

50. Letter from Emily Parker to Emily Hughes, dated November 28th 1938. Friends Service Council, box FSC/R/SP/3/1, Murcia. FHL.

51. Letter from Emily Hughes to Emily Parker, dated December 13th 1938. Friends Service Council, box FSC/R/SP/3/1, Murcia. FHL.

52. Letter from Ruth Cope to Emily Hughes, dated January 24th 1939. Friends Service Council, box FSC/R/SP/3/1, Murcia. FHL.

53. Letter to Edith Pye, dated March 20th 1938. Friends Service Council, box FSC/R/SP/3/1, Murcia. FHL.

54. Dorothy Litten tomó el apellido de su marido al casarse y pasó a llamarse Dorothy Davies.

55. Copy of the report received from Dorothy Litten dated August 2nd, 1938. Friends Service Council, box FSC/R/ $\mathrm{SP} / 3 / 3$, Valencia: letters from. FHL.

56. Copy of the report received from Dorothy Litten dated August 2nd, 1938. Friends Service Council, box FSC/R/ $\mathrm{SP} / 3 / 3$, Valencia: letters from. FHL.

57. Copy of the report received from Dorothy Litten dated August 2nd, 1938. Friends Service Council, box FSC/R/ $\mathrm{SP} / 3 / 3$, Valencia: letters from. FHL.

58. De la Serena, Antonio, "Alicante. Como viven los evacuados de Madrid", Mundo Gráfico, 26 mayo 1937.

59. Davies, Dorothy, Six months in Southern Spain. 1938. Box D-2: AC/1. Marx Memorial Library (Londres).

60. Copy of the report received from Dorothy Litten dated August 2nd, 1938. Friends Service Council, box FSC/R/ $\mathrm{SP} / 3 / 3$, Valencia: letters from. FHL.

61. Davies, Dorothy, Six months in Southern Spain. 1938. Box D-2: AC/1. Marx Memorial Library (Londres). 
62. Letters from Dorothy Morris, a letter from the Hospital in Murcia. 5 enero 1939. MS-Papers-9144. Alexander Turnbull Library, National Library of New Zealand, Wellington (Nueva Zelanda).

63. Archivo de la familia Danjou.

64. Letters from Dorothy Morris, Hotel Regina Perpignan. 2 junio 1939. MS-Papers-9144. Alexander Turnbull Library,

\section{BIBLIOGRAFÍA}

Alted Vigil, Alicia (2003), "Los niños de la Guerra Civil", Anales de Historia Contemporánea, 19, pp. 43-58.

Barona, Josep Lluís; Bernabeu-Mestre, Josep (2008), La salud y el Estado. El movimiento sanitario internacional y la administración española, Valencia, Publicacions de la Universitat de València.

Beneito Lloris, Àngel (2016), “L'ajuda sanitària escandinava durant la Guerra Civil espanyola: de I'Hospital Suec-Noruec d'Alcoi a l'Hospital Militar Base", Afers, 31 (84), pp. 345-363

Bethune, Norman (1937), The Crime on the Road: MálagaAlmería, Madrid, Publicaciones Iberia.

Bethune, Norman (2012), Las heridas, Logroño, Pepitas de calabaza.

Blanc Rodríguez, Manuel (1932), "Tratamiento de la tos ferina por vacunación intradérmica", Archivos españoles de Pediatría, 16 (1), pp. 18-19.

Butler, Paddy (2017), The extraordinary story of Mary Elmes. The Irish Oskar Schindler, Dublín, Orpen Press.

Bosch Marín, Juan; Blanco Otero, Manuel; Mingo de Benito, José María et al. (1949), "La puericultura en la sanidad nacional", Al servicio de España y del niño español, Madrid, Dirección General de Sanidad, Madrid, núm. 142.

Casañ Guillem (2006), "El hospital de Benicàssim en el contexto del servicio sanitario de las Brigadas Internacionales (Guerra Civil, 1936-1939)". En: Requena Gallego, Manuel y Sepúlveda Losa, Rosa María (coords.), La Sanidad en las Brigadas Internacionales, Cuenca, Ediciones de la Universidad de Castilla-La Mancha, pp. 161-198.

Cerro Aguilar, Enrique (2007), Camino de Annual. Albacete y el Desastre de 1921, Albacete, Instituto de estudios albacetenses.

Coni, Nicholas (2008), Medicine and Warfare. Spain, 19361939, Routledge/Cañada Blanch, Nueva York/Londres.

Derby, Mark (2015), Petals and Bullets. Dorothy Morris. New Zealand Nurse in the Spanish Civil War, Brighton, Chicago, Toronto, Sussex Academic Press/Cañada Blanch Centre for Contemporary Spanish Studies.

Farré, Sébastien (2014), Colis de guerre: secours alimentaire et organisations humanitaires (1914-1947), Rennes, Presses Universitaires de Rennes.
National Library of New Zealand, Wellington (Nueva Zelanda).

65. Oficio del alcalde de Alicante al de Cieza. 14 septiembre 1939. Legajo 1902-23-119/0. Arxiu Municipal d'Alacant.

66. Carta de George Young a Miss Thomson. 20 abril 1939. Archivo de la familia Young.

Fernández Soria, Juan Manuel (1987), "La asistencia a la infancia en la Guerra Civil. Las colonias escolares", Revista de Historia de la Educación, 6, pp. 83-128.

Finestres, Jordi (2012), El xalet de Puigcerdà. Solidaritat sota les bombes, Badalona, Ara Llibres.

Finn, Clodahg (2017), A Time to Risk All. The untold story of Mary Elmes, the Irishwoman who saved children from Nazi concentration camps, Dublín, Gill Books.

Fyrth, Jim (1986), The Signal was Spain: The Aid Spain Movement in Britain 1936-39, London, Lawrence \& Wishart.

García Ferrandis, Xavier (2015), L'assistència sanitària a la ciutat de València durant la Guerra Civil, Valencia, Publicacions de la Universitat de València.

García Ferrandis, Xavier; Martínez-Vidal, Àlvar (2017), “Enfermeras británicas en una misión humanitaria cuáquera: el Hospital Inglés de Alicante para niños refugiados durante la Guerra Civil Española”. En: Zarzoso, Alfons y Arrizabalaga, Jon (eds.), Al servicio de la salud humana. La historia de la medicina ante los retos del siglo XXI, Sant Feliu de Guíxols, Sociedad Española de Historia de la Medicina, pp. 195-201.

Jackson, Angela (2010), Las mujeres británicas y la Guerra Civil española,Valencia, Publicacions de la Universitat de València.

Jiménez Lucena, Isabel (1994), “El tifus exantemático de la posguerra española (1939-1943). El uso de una enfermedad colectiva en la legitimazión del "Nuevo Estado»", Dynamis, 24, pp. $185-198$

Keene, Judith (2001), Fighting for Franco. International Volunteers in the Nationalist Spain during the Spanish Civil War, Londres, Hambledon Continuum

Kershner, Howard E. (2011), La labor asistencial de los cuáqueros durante la Guerra Civil española y la posguerra. España y Francia 1936-1941, Madrid, Siddharth Mehta Ediciones, pp. 7-15.

López Vallecillo, María (2016), Presencia social e imagen pública de las enfermeras en el siglo XX (1915-1940), Universidad de Valladolid.

Majada Neila, Jesús (2014), Norman Bethune: la huella solidaria, Madrid, Centro Cultural de China en Madrid.

Martín Moruno, Dolores; Ordóñez Rodríguez, Javier (2009), "The nursing vocation as political participation for women 
during the Spanish Civil War", Journal of War and Culture, 2 (3), pp. 305-319.

Martín Moruno, Dolores (2010), "Becoming visible and real: Images of Republican Women during the Spanish Civil War", Visual Culture \& Gender, 5, pp. 5-15.

Martín Moruno, Dolores (2017), "Tejiendo redes de cuidados: la compasión como conocimiento de las mujeres humanitarias en la guerra (1853-1945)". En: Zarzoso, Alfons y Arrizabalaga, Jon (eds.), Al servicio de la salud humana. La historia de la medicina ante los retos del siglo XXI, Sant Feliu de Guíxols, Sociedad Española de Historia de la Medicina, pp. 21-26.

Martorell, Manuel (2000), Jesús Monzón: el líder comunista olvidado por la historia, Arre (Navarra), Pamiela, 2000.

Mendlesohn, Farah (2002), Quaker relief work in the Spanish Civil War, Nueva York, The Edwin Mellen Press.

Palfreeman, Linda (2014), Aristocrats, Adventurers and Ambulances: British Ambulance Units in the Spanish Civil War, Brighton, Chicago, Toronto, Sussex Academic Press/Cañada Blanch Centre for Contemporary Spanish Studies, pp. 130-159.

Palfreeman, Linda (2016), "Assistència sanitària a la rereguarda de Madrid: els hospitals britànics de Huete i Uclés", Afers, 31 (84), pp. 305-326.

Perdiguero-Gil, Enrique; Bernabeu-Mestre, Josep (1999), "La Gota de Leche de Alicante (1925-1940)". En: Benito Lloris, Àngel; Blay Meseguer, Francesc Xavier y Lloret Pastor, Joan (eds.), Beneficència i Sanitat en els municipis valencians, Alcoi, Centre Alcoià d'Estudis Històrics i Arqueològics, pp. 291-310.

Perdiguero-Gil, Enrique; Bernabeu-Mestre, Josep (2005), “Child Care in Urban and Rural Alicante: the Gota de Leche". En: Barona, Josep Lluis y Cherry Steven (eds.), Health and Me- dicine in Rural Europe (1850-1945), Valencia, Seminari d'Estudis sobre la Ciència, pp. 347-359.

Pretus, Gabriel (2015), La ayuda humanitaria de en la Guerra Civil española (1936-1939), Granada, Comares.

Puerta Jiménez, Inmaculada; Sáez Gómez, José Miguel; Aguinaga Ontoso, Enrique (2015), La Sociedad de Medicina y Cirugía de Levante a través de sus publicaciones: el papel minoritario del laboratorio y la salud pública, Alcoi, 3Ciencias.

Rodríguez-Ocaña, Esteban (1996) “Una medicina para los niños". En Borrás Llop, José María (ed.), Historia de la infancia en la España contemporánea, Madrid, Ministerio de Trabajo y Asuntos Sociales, pp. 149-169.

Serra Sala, Rosa (2006), L'ajuda humanitària dels quàquers als infants de Catalunya durant la Guerra Civil, 1936/1939, Universitat de Girona.

Serrallonga i Urquidi, Joan (2007), "El aparato provincial durante la Segunda República. Los gobernadores civiles, 19311939", Hispania Nova. Revista de Historia Contemporánea, 7, $7 \mathrm{a} 008$.

Torró Martínez, Juan José (2016), “Solidaritat socialista i ajuda mèdica a la causa republicana durant la Guerra Civil (19361939): I'Hospital Militar Internacional d'Ontinyent", Afers, 31 (84), pp.327-344.

Van Etten, Henry (1938), Le Secours Quaker International, París, Société des Amis, pp. 11-12.

Wilson, Francesca (1944), In the Margins of Chaos. Recollections of Relief Work in and between Three Wars, London, John Murray.

Wilson, Bernard (2012), "Mary Elmes 1908-2002", Toulouse Quakers Weblog, [en línea], disponible en: https://toulousequakers.wordpress.com/2012/04/28/mary-elmes-1908-2002/, [consultado el 02/03/2017] 